\title{
Lung fibrosis as a potential complication of the hemostatic tissue sealant, biologic glue (Bioglue)
}

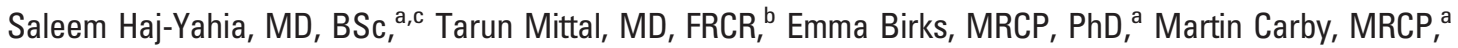 \\ Mario Petrou, FRCS, PhD, ${ }^{a}$ John Pepper, FRCS, ${ }^{a}$ Gilles Dreyfus, MD, PhD, a and Mohammed Amrani, MD, PhD, ${ }^{a}$ London, UK
}

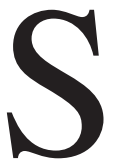

urgical use of the hemostatic tissue sealant Bioglue has become a widely accepted and effective method to control postoperative bleeding and airway air leak (AAL). ${ }^{1,2}$ However, recently emerging evidence of potential complications and hazards that arise from its extensive use has raised concerns about its clinical safety. ${ }^{3}$

\section{Clinical Summary}

A 27-year-old woman with acute viral myocarditis had severe heart failure, sepsis, multiorgan failure, and acute hemodynamic deterioration despite maximum medical therapy. A salvage Levitronix left ventricular assist device (LVAD) was urgently implanted as a "bridge to decision" until the reversibility of her condition could be clarified. However, due to poor right ventricular functioning, a Centrimag (Levitronix, Zurich, Switzerland) right ventricular assist device (RVAD) had to be implanted 3 days later. Following an apparently good recovery after 2 weeks, the Levitronix LVAD was upgraded to a Jarvik 2000 ventricular assist device (Jarvik Heart, Inc, New York, NY) for longer-term support; the RVAD was left in place due to poor right ventricular function. The initial postoperative course was turbulent, complicated by 2 sternal reexplorations for significant diffuse bleeding from the surface of the right lung and chest wall (the left lung and pleura were kept intact), which could not be controlled with diathermy or pharmacologic agents. A thick 5-pack layer of Bioglue sealant was applied over the surface of the right lung and the corresponding right chest wall to control the bleeding. Six days later, the patient had sepsis and her hemoglobin level dropped; the chest radiographs performed at this time showed progressive complete opacification of the right hemithorax (Figure 1, A). A computed tomography (CT) scan of the chest demonstrated a loculated fluid/blood collection in the pleural space extending all along the chest wall and into the

From the Departments of Cardiothoracic Transplantation and Mechanical Circulatory Support ${ }^{\mathrm{a}}$ and Radiology, ${ }^{\mathrm{b}}$ Royal Brompton \& Harefield Hospital, NHS Trust, London, UK; and National Heart \& Lung Institute, ${ }^{\mathrm{C}}$ Imperial College London, London, UK

Cardiothoracic Surgery, Transplantation \& Mechanical Circulatory Support, Royal Brompton \& Harefield Hospital, London, UK.

Received for publication Oct 6, 2006; accepted for publication Nov 28, 2006.

Address for reprints: Saleem Haj-Yahia, MD, BSc, Royal Brompton \& Harefield NHS trust, Cardiothoracic Transplantation and Mechanical Circulatory Support, Hill End Road Harefield, London UB9 6RJ, UK (E-mail: s.haj-yahia@imperial.ac.uk).

J Thorac Cardiovasc Surg 2007;133:1387-8

$0022-5223 / \$ 32.00$

Copyright $\odot 2007$ by The American Association for Thoracic Surgery doi:10.1016/j.jtcvs.2006.11.072 oblique fissure (but no intrapleural bleed was noted) (Figure 1, B), for which she needed another resternotomy, and Bioglue was applied again to control the bleeding. Subsequently, her condition started to improve, and she was weaned off the ventilator and hemofiltration; her kidney and liver functions normalized and were cleared of infection. Radiologic findings after the surgery, both chest radiographs and $\mathrm{CT}$ scans after the evacuation of the clot, continued to show minor pleural collection, and, more importantly, changes of patchy pulmonary fibrosis in the right lung with other areas of segmental collapse (Figure 2, $A$ and $B$ ). Multiple assessments of the heart at a later stage showed no evidence of ventricular recovery. Therefore, she was listed and urgently underwent a transplant after 101 days of mechanical circulatory support. During the surgery, the right pleural space was fully obliterated and severe dense adhesions were noted with a thick layer of Bioglue. The post-
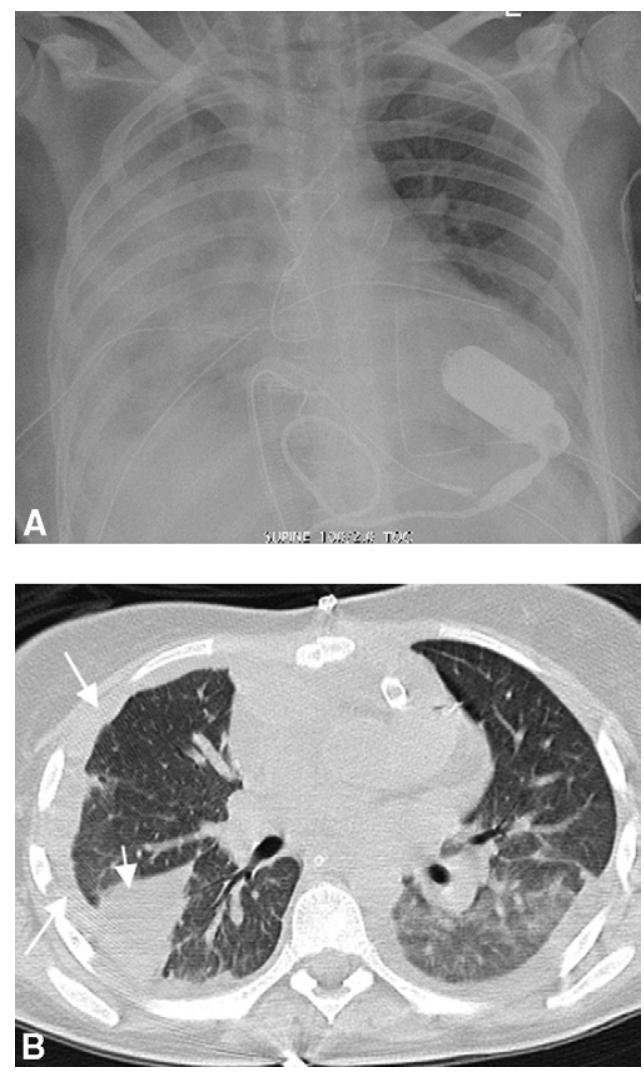

Figure 1. A, A radiograph showing the progressive complete opacification of the right hemithorax due to active postoperative bleeding. B, CT scan of the chest on the first postoperative day showing a loculated blood collection in the pleural space. 

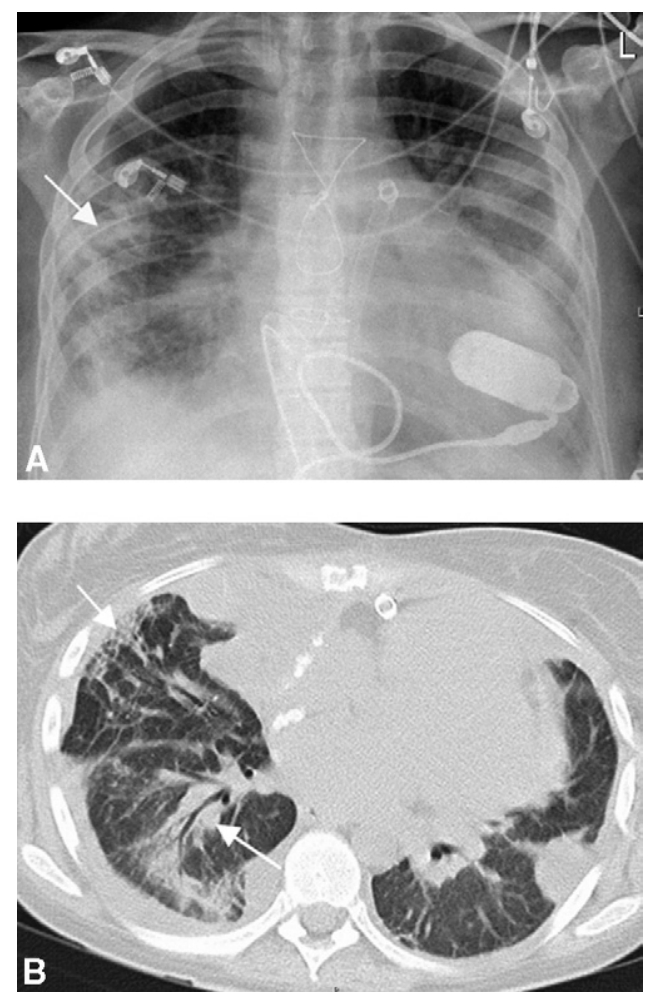

Figure 2. A, A radiograph showing patchy pulmonary fibrosis following the application of Bioglue. B, A CT scan showing patchy pulmonary fibrosis in the right lung with other areas of segmental collapse following the application of Bioglue. These fibrotic changes remained persistent on later radiologic follow.

operative course was uneventful and she was discharged home 42 days later. On later radiologic follow-up, the right lung remained expanded but with persistent fibrosis.

\section{Discussion}

Bioglue, a 2-component tissue sealant composed of bovine serum albumin and glutaraldehyde, has been proven effective in controlling bleeding after major cardiovascular surgery and in controlling AAL following lung surgery. ${ }^{1,2}$ Along with its occasionally life- saving properties, a number of cautionary notes have recently been reported regarding potential complications associated with its use. ${ }^{3}$ Some of these complications related to its quick and robust sealant and glue properties (such as acute intraoperative malfunction of the aortic valve, Bioglue coronary embolism, aseptic mediastinal cyst, and so on). However, other complications are related to its compound biochemical properties. In vivo studies indicated that polymerized Bioglue releases amounts of glutaraldehyde that are capable of inducing cytotoxic effects. ${ }^{4}$ It has been shown that lungs and liver tissue are particularly sensitive to its toxic effect; therefore, Furst and Banerjee ${ }^{4}$ concluded that its use should be restricted to aortic dissection only. Erasmi ${ }^{5}$ has described the histopathologic findings in aortic specimens treated with Bioglue as "severe inflammation with multiple-granulocytes and histiocytes and a massive foreign-body reaction with numerous multinucleated giant cells." However, Bioglue has so far been widely used and is considered safe and effective in lung surgery as a sealant to prevent AAL, possibly because it has been used in small amounts and in a controlled fashion on restricted areas. In our experience, we used Bioglue in large amounts over the surface of the lung to control severe diffuse bleeding, which was impossible to control using other pharmacologic or surgical means and proved to be a lifesaving procedure in this case. However, we feel that the large amount used in our case is probably responsible for the fibrotic changes that occurred.

In conclusion, the use of Bioglue should be kept to the minimum amount needed to achieve hemostasis or to control AAL, and it can be considered particularly detrimental when used on lung tissue.

\section{References}

1. Raanani E, Latter DA, Errett LE, Bonneau DB, Leclerc Y, Salasidis GC Use of "BioGlue" in aortic surgical repair. Ann Thorac Surg. 2001;72: 638-40.

2. Tansley P, Al-Mulhim F, Lim E, Ladas E, Goldstraw P. A prospective, randomized, controlled trial of the effectiveness of BioGlue in treating alveolar air leaks. J Thorac Cardiovasc Surg. 2006;132:105-12.

3. Ngaage DL, Edwards WD, Bell MR, Sundt TM. A cautionary note regarding long- term sequelae of biologic glue. J Thorac Cardiovasc Surg. 2005;129:937-8.

4. Furst W, Banerjee A. Release of glutaraldehyde from an albuminglutaraldehyde tissue adhesive causes significant in vitro and in vivo toxicity. Ann Thorac Surg. 2005;79:1522-9.

5. Erasmi AW, Sievers $\mathrm{HH}$, Wolschlager C. Inflammatory response after BioGlue application. Ann Thorac Surg. 2002;73:1025-6. 